\title{
Discovery of optical candidate supernova remnants in Sagittarius
}

\author{
J. Alikakos ${ }^{1,2}$, P. Boumis ${ }^{1}$, P. E. Christopoulou ${ }^{2}$, and C. D. Goudis ${ }^{1,2}$ \\ ${ }^{1}$ Institute of Astronomy \& Astrophysics`, National Observatory of Athens, I. Metaxa \& V. Pavlou, P. Penteli, 15236 Athens, Greece \\ e-mail: [johnal; ptb; cgoudis] @astro.noa.gr \\ 2 Astronomical Laboratory, Department of Physics, University of Patras, 26500 Rio-Patras, Greece \\ e-mail: pechris@upatras.physics.gr
}

Received 27 February 2012 / Accepted 4 July 2012

\begin{abstract}
During an [O III] survey of planetary nebulae, we identified a region in Sagittarius containing several candidate supernova remants (SNRs) and obtained deep optical narrow-band images and spectra to explore their nature. We obtained images of the area of interest by acquiring observations in the emission lines of $\left.\mathrm{H} \alpha+\left[\mathrm{N}_{\mathrm{II}}\right], \mathrm{S}_{\mathrm{II}}\right]$ and $\left[\mathrm{O}_{\mathrm{III}}\right]$. The resulting mosaic covers an area of $1.4^{\circ} \times 1.0^{\circ}$, where both filamentary and diffuse emission was discovered, suggesting that there is more than one SNR in the area. Deep long-slit spectra were also taken of eight different regions. Both the flux-calibrated images and the spectra show that the emission from the filamentary structures originates from shock-heated gas, while the photo-ionization mechanism is responsible for the diffuse emission. Part of the optical emission is found to be correlated with the radio at $4850 \mathrm{MHz}$ suggesting that they are related, while the infrared emission found in the area at $12 \mu \mathrm{m}$ and $22 \mu \mathrm{m}$ marginally correlates with the optical. The presence of the [O III] emission line in one of the candidate SNRs implies that the shock velocities in the interstellar "clouds" are between $120 \mathrm{~km} \mathrm{~s}^{-1}$ and $200 \mathrm{~km} \mathrm{~s}^{-1}$, while its absence in the other candidate SNRs indicates that the shock velocities there are slower. For all candidate remnants, the [S II] $\lambda \lambda 6716 / 6731$ ratio indicates that the electron densities are below $240 \mathrm{~cm}^{-3}$, while the $\mathrm{H} \alpha$ emission is measured to be between 0.6 and $41 \times 10^{-17} \mathrm{erg} \mathrm{s}^{-1} \mathrm{~cm}^{-2} \operatorname{arcsec}^{-2}$. The existence of eight pulsars within $1.5^{\circ}$ of the center of the candidate SNRs also implies that there are many SNRs in the area as well as that the detected optical emission could be part of a number of supernovae explosions.
\end{abstract}

Key words. ISM: general - ISM: supernova remnants

\section{Introduction}

Supernova explosions belong to the most spectacular events in the Universe. Observations of galaxies reveal that several events occur every year (Mannucci et al. 2005), where the supernova is of comparable brightness to the entire galaxy for between days and weeks. Supernova remnants (SNRs), which are the consequent results of these events, are among the strongest radio sources observed. They have a major influence on both the properties of the interstellar medium (ISM) and the evolution of galaxies as a whole. They enrich the ISM with heavy elements, release about $10^{51}$ ergs of energy, heat the ISM, compress the magnetic field, and efficiently accelerate, in their shock waves, energetic cosmic rays observed throughout the Galaxy. The majority of known SNRs have been discovered based on their non-thermal radio emission (Green 2009), while a smaller number of them have been observed at other wavelengths (e.g. optical: Boumis et al. 2008, 2009; Stupar \& Parker 2011; X-rays: Reynolds et al. 2009; and infrared: Reach et al. 2006).

In this paper, we report the optical detection of many filamentary and diffuse structures (possibly more than one SNR) in the region of the Sagittarius constellation. During an [O III] $5007 \AA$ survey of planetary nebulae (Boumis et al. 2003, 2006), we identified a very strong [ $\mathrm{S}_{\mathrm{II}}$ ] source designated as a candidate SNR instead of a planetary nebula. Following that detection, a number of images in $\mathrm{H} \alpha+\left[\mathrm{N}_{\mathrm{II}}\right]$, [S II $]$, and [O III]

\footnotetext{
* Renamed to: Institute of Astronomy, Astrophysics, Space Applications and Remote Sensing.
}

were taken to explore the SNR candidate area and many filamentary structures were discovered. Only one known SNR was found in the area (G 16.2-2.7; Trushkin 1999), hence all the other filamentary structures were examined in detail to identify their origin. Information about the observations and data reduction is given in Sect. 2. In Sects. 3 and 4, the results of the imaging and spectra observations are presented, while in Sect. 5 we report on observations at wavelengths other than the optical. Finally, in Sect. 6 we discuss the properties of the new candidate SNRs.

\section{Observations}

A summary and log of our observations are given in Table 1. In the sections below, we describe these observations in detail.

\subsection{Imaging}

All images were taken with the $0.3 \mathrm{~m}$ Schmidt-Cassegrain $(f / 3.2)$ telescope at Skinakas Observatory in Crete, Greece, in 2005, on June 7, 8, and 9, and August 26 and 28. A $1024 \times$ 1024 Thomson CCD was used, which has a pixel size of $19 \mu \mathrm{m}$ resulting in a $70^{\prime} \times 70^{\prime}$ field of view and an image scale of $4^{\prime \prime}$ pixel $^{-1}$. The area of interest was observed for $2400 \mathrm{~s}$ in $\mathrm{H} \alpha+[\mathrm{N}$ II $],\left[\mathrm{S} \mathrm{II}_{\mathrm{II}}\right.$ and [O III] filters, while corresponding continuum images were also observed (180 s each); afterwards, the appropriate scaling were subtracted from the narrow-band images to eliminate the confusing star field. The continuum-subtracted 
Table 1. Imaging and spectral log.

\begin{tabular}{|c|c|c|c|c|}
\hline \multicolumn{5}{|c|}{ Imaging } \\
\hline Filter & $\begin{array}{l}\lambda_{\mathrm{c}} \\
(\AA) \\
\end{array}$ & $\begin{array}{l}\Delta \lambda \\
(\AA)\end{array}$ & $\begin{array}{l}\text { Total exp. time } \\
\text { (s) }\end{array}$ & Telescope \\
\hline $\mathrm{H} \alpha+\left[\mathrm{N}_{\text {II }}\right] 6548 \& 6584 \AA$ & 6570 & 75 & $4800(2)^{a}$ & $0.3 \mathrm{~m}$ Skinakas \\
\hline [O III] $5007 \AA$ & 5005 & 28 & $4800(2)$ & $0.3 \mathrm{~m}$ Skinakas \\
\hline [S II] $6716 \& 6731 \AA$ & 6720 & 18 & $4800(2)$ & $0.3 \mathrm{~m}$ Skinakas \\
\hline Cont blue & 5470 & 230 & 180 & $0.3 \mathrm{~m}$ Skinakas \\
\hline Cont red & 6096 & 134 & 180 & $0.3 \mathrm{~m}$ Skinakas \\
\hline $\mathrm{H} \alpha+\left[\mathrm{N}_{\text {II }}\right] 6548 \& 6584 \AA$ & & 80 & 10800 & $1.2 \mathrm{~m} \mathrm{AAO/UKST}$ \\
\hline \multicolumn{5}{|c|}{ Spectroscopy } \\
\hline \multirow[t]{2}{*}{ Slit position } & \multicolumn{2}{|c|}{ Slit centers } & Offset $^{b}$ & Aperture length $^{c}$ \\
\hline & $\begin{array}{c}\alpha \\
(\mathrm{h} \mathrm{m} \mathrm{s})\end{array}$ & $\begin{array}{c}\delta \\
\left(0^{\prime}, \prime\right)\end{array}$ & $(\operatorname{arcsec})$ & $(\operatorname{arcsec})$ \\
\hline $1 \mathrm{a}$ & 182909 & -163132 & $2.4 \mathrm{~N}$ & 10.6 \\
\hline $1 b$ & 182909 & -163132 & $28.9 \mathrm{~S}$ & 18.9 \\
\hline $2 \mathrm{a}$ & 182858 & -163600 & $95.0 \mathrm{~N}$ & 26.0 \\
\hline $2 b$ & 182858 & -163600 & $70.2 \mathrm{~N}$ & 11.8 \\
\hline $2 c$ & 182858 & -163600 & $19.5 \mathrm{~N}$ & 13.0 \\
\hline $3 a$ & 182849 & -162455 & $137.5 \mathrm{~N}$ & 27.1 \\
\hline $3 b$ & 182849 & -162455 & $33.6 \mathrm{~N}$ & 43.6 \\
\hline $3 c$ & 182849 & -162455 & $20.1 \mathrm{~S}$ & 23.6 \\
\hline 4 & 182955 & -163412 & $16.6 \mathrm{~S}$ & 30.7 \\
\hline 5 & 182842 & -161502 & $37.2 \mathrm{~S}$ & 60.2 \\
\hline $6 a$ & 182741 & -161739 & $74.3 \mathrm{~S}$ & 30.7 \\
\hline $6 b$ & 182741 & -161739 & $116.2 \mathrm{~S}$ & 20.0 \\
\hline 7 & 182726 & -160750 & $32.4 \mathrm{~N}$ & 31.9 \\
\hline 8 & 182612 & -160158 & $27.7 \mathrm{~N}$ & 23.6 \\
\hline
\end{tabular}

Notes. ${ }^{(a)}$ Numbers in parentheses represent the number of individual frames. ${ }^{(b)}$ Spatial offset from the slit center in arcsec: $\mathrm{N}(=\mathrm{North}), \mathrm{S}(=\mathrm{South})$. (c) Aperture lengths for each area in arcsec.

images of the $\mathrm{H} \alpha+\left[\mathrm{N}\right.$ II] and $\left[\mathrm{S}_{\mathrm{II}}\right]$ emission lines are shown in Figs. 1 and 2.

The image reduction was carried out using the $\operatorname{IRAF}^{1}$ and MIDAS $^{2}$. All frames were bias-subtracted and flat-field corrected using a series of twilight flat-fields. The absolute flux calibration was performed through observations of a series of spectrophotometric standard stars (HR 5501, HR 7596, HR 7950, HR 8634, and HR 9087; Hamuy et al. 1992). The astrometric solution for all data frames was calculated using the Space Telescope Science Institute (STScI) Guide Star Catalogue II (GSC-II; Lasker et al. 2008). All the equatorial coordinates quoted in this work, refer to epoch 2000.

To cover in full the area of interest, we used wide-field images from the SuperCOSMOS H $\alpha$ Survey (SHS; Parker et al. 2005). The resulting $1.4^{\circ} \times 1.0^{\circ}$ mosaic was created from 16 different fields, each one having a $30^{\prime} \times 30^{\prime}$ field of view and an image scale of $0.67^{\prime \prime}$ pixel $^{-1}$. The mosaic was used to compare the detected optical emission with other wavebands (radio and infrared - see Sect. 3.3 and Figs. 5, 6). The details of all imaging observations are given in Table 1 .

\subsection{Spectroscopy}

Low-dispersion, long-slit spectra were obtained with the $1.3 \mathrm{~m}$ Ritchey-Cretien ( $f / 7.7)$ telescope at Skinakas Observatory in 2005 on June 4 and 5, July 10, and September 6 and 7.

\footnotetext{
1 Image Reduction and Analysis Facility (http://iraf.noao.edu/).

2 Munich Image Data Analysis System

(http://www.eso.org/sci/software/esomidas/).
}

The 1300 line $\mathrm{mm}^{-1}$ grating was used together with a $2000 \times$ 800 SITe CCD $\left(15 \times 15 \mu \mathrm{m}^{2}\right.$ pixels $)$, resulting in a scale of $1 \AA$ pixel $^{-1}$ and covering the range 4750-6815. . The above combination results in a spectral resolution of $\sim 8 \AA$, and $\sim 11 \AA$ at red and blue wavelengths, respectively. The slit width was 7'.7 and its length 7!9, and in all cases it was oriented in the southnorth direction. The spectrophotometric standard stars HR 4468, HR 5501, HR 7596, HR 9087, HR 8634, and HR 7950 (Hamuy et al. 1992) were observed to calibrate the spectra. The data reduction was performed using the IRAF package (twodspec).

Deep low-resolution spectra were taken of the relatively bright optical filament (their exact positions are given in Table 1). In Table 4, we present the relative line fluxes taken from different apertures ( $a, b$, and c) along each slit. In particular, apertures a, b, and c correspond to offset positions (see Table 1) north or south of the slit center, which were selected because they are free of field stars in an otherwise crowded field and include sufficient line emission to permit an accurate determination of the observed line fluxes (their exact aperture length is given in Table 1). The background extraction aperture was taken towards either the northern or southern end of the slits depending on the slit position. The signal-to-noise ratios ( $\mathrm{S} / \mathrm{N}$ 's) presented in Table 4 do not include calibration errors, which are smaller than 10 percent. Typical spectra are shown in Fig. 3.

\section{Results}

\subsection{The optical emission-line images}

The images in Figs. 1 and 2 show a considerable amount of new faint optical emission including filamentary and diffuse 


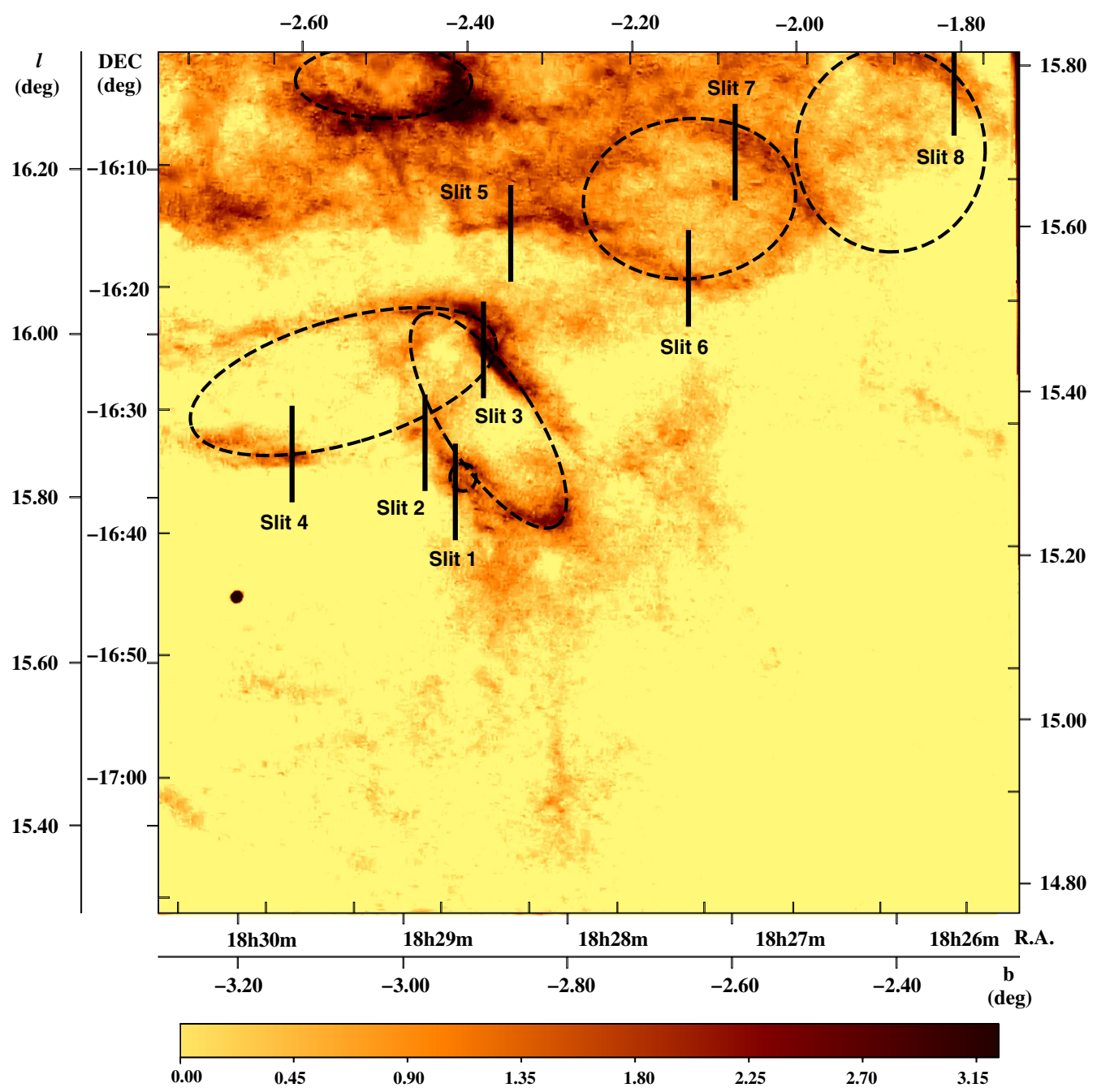

Fig. 1. The continuum-subtracted image of the observed area in Sagittarius, in $\mathrm{H} \alpha+\left[\mathrm{N}_{\mathrm{II}}\right]$ emission in both equatorial (RA, Dec internal lines on axis) and Galactic (l, b external lines on axis) coordinates. The black lines indicate the positions of the slits and the dashed-ellipses the geometry of the newly discovered candidate SNRs. Shadings run linearly from 0 to $3.2 \times 10^{-17} \mathrm{erg} \mathrm{s}^{-1} \mathrm{~cm}^{-2} \operatorname{arcsec}^{-2}$.

structures. The $\mathrm{H} \alpha+\left[\mathrm{N}_{\text {II }}\right]$ (Fig. 1) image contains the clearest evidence of the newly detected structures, while the [S II] image (Fig. 2) also shows strong emission but less filamentary structure. In contrast, $[\mathrm{O} \mathrm{III}]$ emission was only detected in one small area and is not shown here.

Since all images are flux-calibrated, they provide a first indication of the nature of the observed emission (see Table 2). A study of these images shows that most parts of the optical emission originate from shock-heated gas since we estimate ratios $[\mathrm{S}$ II $] / \mathrm{H} \alpha \geq 0.47$. This conclusion is verified by the deep long-slit spectra that yield more accurate measurements of the individual line fluxes. The variety of structures detected in the $\mathrm{H} \alpha+\left[\mathrm{N}_{\text {II }}\right]$ and $\left[\mathrm{S}_{\mathrm{II}}\right]$ images are not present in the medium ionization line of [O III], apart from one area (slit 1), hence the $3 \sigma$ upper limit over the area given in Table 2.

Several thin and curved filaments can be seen in Fig. 1. In general, the field appears somewhat complex owing to the many filamentary structures and significant number of diffuse emission structures. A search in the database did not reveal any known bright optical nebula. A combination of their morphology and the spectroscopic results suggests that there is more than one possible SNR in the region. In particular, following their geometry we suggest that there is six candidate SNRs (indicated as dashed-ellipses in Fig. 1). Their names, centers, and diameters are presented in Table 3. The possibility of fewer or more SNRs in the region cannot of course be ruled out.

The most interesting filaments of the candidate SNRs are described here in more detail.

$G$ 15.6-2.6. The basic characteristic of its filaments is their brightness in $\mathrm{H} \alpha+\left[\mathrm{N}_{\mathrm{II}}\right]$ (Fig. 1) and [ $\left.\mathrm{S}_{\mathrm{II}}\right]$ (Fig. 2). The bulk of the emission seems to be bounded by at least two very bright complex filaments. The southern structure covers the area from $\delta \sim-16^{\circ} 39^{\prime}, \alpha \simeq 18^{\mathrm{h}} 28^{\mathrm{m}} 21^{\mathrm{s}}$ to $\delta \sim-16^{\circ} 33^{\prime}$, $\alpha \simeq 18^{\mathrm{h}} 29^{\mathrm{m}} 01^{\mathrm{s}}$, while the northern structure, having the same inclination $\left(\sim 45^{\circ}\right)$, with respect to the east-west direction, covers the area between $\delta \sim-16^{\circ} 29^{\prime}, \alpha \simeq 18^{\mathrm{h}} 28^{\mathrm{m}} 31^{\mathrm{s}}$ and $\delta \sim-16^{\circ} 21^{\prime}$, $\alpha \simeq 18^{\mathrm{h}} 28^{\mathrm{m}} 55^{\mathrm{s}}$. No significant emission was found in the image of the [O III] medium ionization line. The morphology of the [S II] image is generally similar to, though not as bright as, that of the $\mathrm{H} \alpha+\left[\mathrm{N}_{\text {III }}\right]$ image. We detected [S II] emission where most of the $\mathrm{H} \alpha+\left[\mathrm{N}_{\mathrm{II}}\right]$ emission was found with filamentary bright structures in the south-east and north-west areas, while diffuse emission characterizes the rest of the remnant's emission. Diffuse emission and several shorter filamentary structures are detected between the north and south boundaries. The images show $\left[\mathrm{S}_{\mathrm{II}}\right] / \mathrm{H} \alpha \geq 0.7$ in agreement with the two spectra positions (slits $1 \& 3$ ), for which $\left[\mathrm{S}_{\mathrm{II}}\right] / \mathrm{H} \alpha \geq 1.0$. We note that some of the filaments to the south and the north seem to correlate with the 


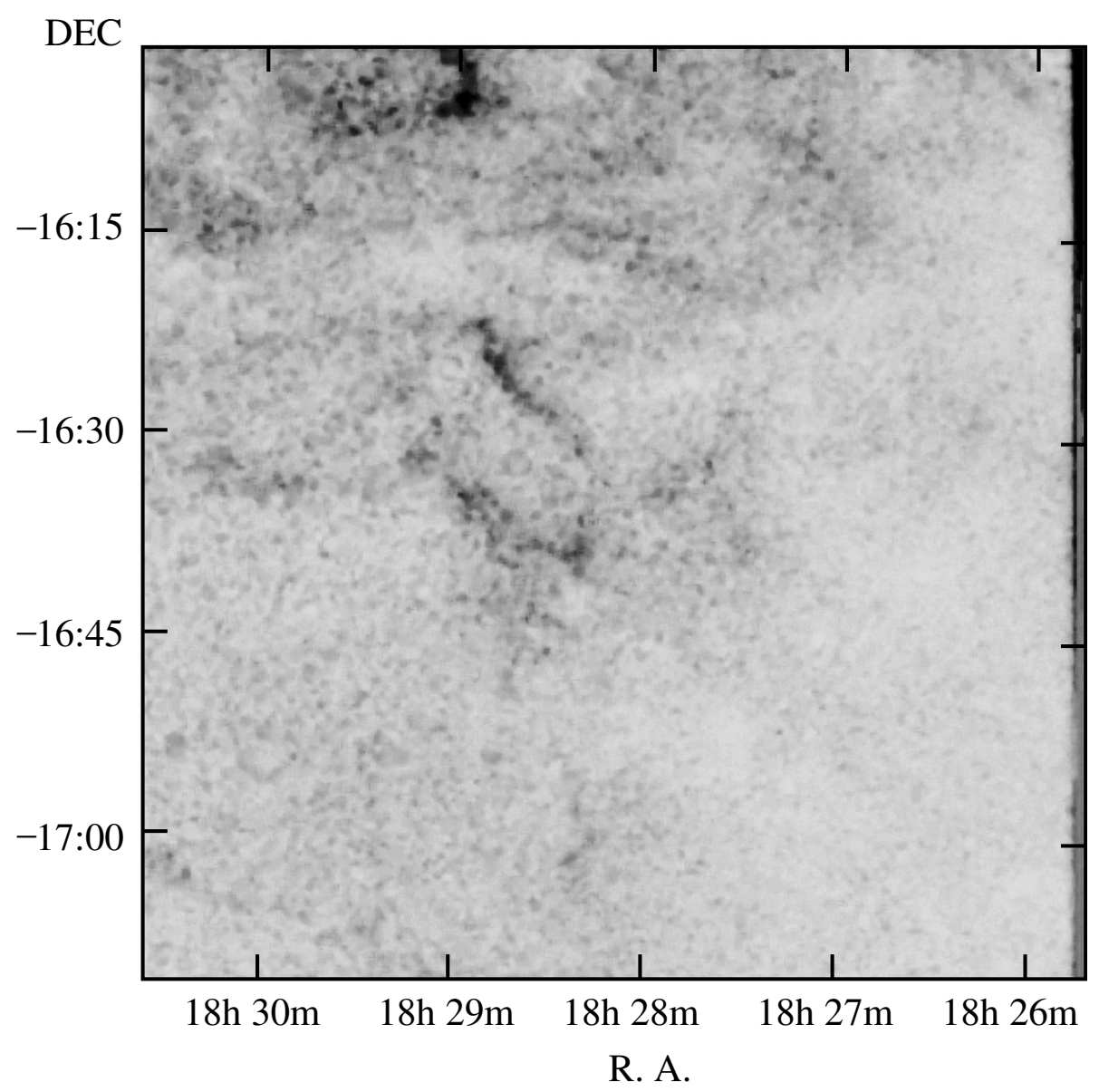

Fig. 2. The continuum-subtracted image of the observed area in $\left[\mathrm{S} \mathrm{II}_{\mathrm{II}}\right.$ emission. Shadings run linearly from 0 to $90 \times 10^{-17} \mathrm{erg} \mathrm{s}^{-1} \mathrm{~cm}^{-2} \operatorname{arcsec}{ }^{-2}$.

Table 2. Typically fluxes measured over the brightest filaments.

\begin{tabular}{lcccccccc}
\hline \hline & Slit 1 & Slit 2 & Slit 3 & Slit 4 & Slit 5 & Slit 6 & Slit 7 & Slit 8 \\
\hline $\mathrm{H} \alpha+[\mathrm{N}$ II $]$ & 42.8 & 62.8 & 114.3 & 64.1 & 67.2 & 50.4 & 57.9 & 53.8 \\
{$\left[\mathrm{~S}_{\mathrm{II}}\right]$} & 19.5 & 18.8 & 40.0 & 16.6 & 15.8 & 15.6 & 17.1 & 13.5 \\
{$\left[\mathrm{O}_{\mathrm{III}}\right]$} & 236 & & & & $<45^{a}$ & & & \\
{$\left[\mathrm{~S}_{\mathrm{II}}\right] / \mathrm{H} \alpha^{b}$} & 0.91 & 0.60 & 0.70 & 0.52 & 0.47 & 0.62 & 0.59 & 0.50 \\
\hline
\end{tabular}

Notes. Fluxes in units of $10^{-17} \mathrm{erg} \mathrm{s}^{-1} \mathrm{~cm}^{-2} \operatorname{arcsec}^{-2}$. Median values over a $40^{\prime \prime} \times 40^{\prime \prime}$ box from selected areas of the bright filaments. ${ }^{(a)} 3 \sigma$ upper limit. ${ }^{(b)}$ The $[\mathrm{N} \mathrm{II}]$ contribution has been removed by using the spectroscopic results.

radio and infrared emission but their resolution does not permit us to verify whether there is a correlation (see Sect. 3.3). New pointed radio observations would be needed to come to a more secure conclusion.

$G$ 15.8-2.8. The most interesting regions lie to the south-east and north-west, where bright filamentary structures are present (between $\alpha \simeq 18^{\mathrm{h}} 29^{\mathrm{m}} 45^{\mathrm{s}}, \delta \simeq-16^{\circ} 34^{\prime} 20^{\prime \prime} ; \alpha \simeq 18^{\mathrm{h}} 30^{\mathrm{m}} 26^{\mathrm{s}}$, $\delta \simeq-16^{\circ} 32^{\prime} 17^{\prime \prime}$ and $\alpha \simeq 18^{\mathrm{h}} 28^{\mathrm{m}} 48^{\mathrm{s}}, \delta \simeq-16^{\circ} 22^{\prime} 45^{\prime \prime}$; $\left.\alpha \simeq 18^{\mathrm{h}} 28^{\mathrm{m}} 58^{\mathrm{s}}, \delta \simeq-16^{\circ} 21^{\prime} 15^{\prime \prime}\right)$. There are also fainter filaments and diffuse emission that cover most of the suggested SNR area, both to the north (from the bright north-west filament to $\alpha \simeq 18^{\mathrm{h}} 30^{\mathrm{m}} 33^{\mathrm{s}}, \delta \simeq-16^{\circ} 25^{\prime} 00^{\prime \prime}$ ) and south (from the bright south-east filament to $\alpha \simeq 18^{\mathrm{h}} 28^{\mathrm{m}} 47^{\mathrm{s}}, \delta \simeq-16^{\circ} 25^{\prime} 30^{\prime \prime}$ ). As for the previous candidate SNR, no [O III] emission was detected. Both images and spectra (slits $2 \& 4$ ) have similar values of $\left[\mathrm{S}_{\mathrm{II}}\right] / \mathrm{H} \alpha$ (between 0.47 and 0.60 ). Part of this SNR to the west overlaps with the north filament of the previous SNR, although their morphology and curvature suggest that the filamentary structures are separated and probably do not correlate with each other. In addition, there is a gap of $\sim 1^{\prime}$ between the very bright filaments which both show infrared emission, while it seems that only the filament from this candidate SNR correlates with the radio emission (Sect. 3.3). However, owing to the low resolution of the radio observations, the possibility that the radio emission comes from both SNRs cannot be ruled out. It should be noted that because the radio emission is generally weak, the overlap of the two remnants would certaintly make it brighter. In addition, the fact that the high-frequency radio emission correlates better with the optical emission than the low-frequency radio emission, which suggests that there is a flatter radio spectrum and therefore thermal radio emission, should be examined further by new pointed radio observations to verify their nature.

$G$ 15.8-2.2. The faint filamentary and diffuse emission of this candidate SNR forms a well-defined ellipse (Fig. 1). There is a bright structure to the east $\left(\alpha \simeq 18^{\mathrm{h}} 28^{\mathrm{m}} 20^{\mathrm{s}}, \delta \simeq-16^{\circ} 14^{\prime} 25^{\prime \prime}\right)$ and many small filaments all around the SNR's borders. The $[\mathrm{S}$ II] $/ \mathrm{H} \alpha$ emission suggests that there are shock-heated mechanisms $(\sim 0.6)$ in agreement in value with that derived from the 
J. Alikakos et al.: Discovery of optical candidate supernova remnants in Sagittarius
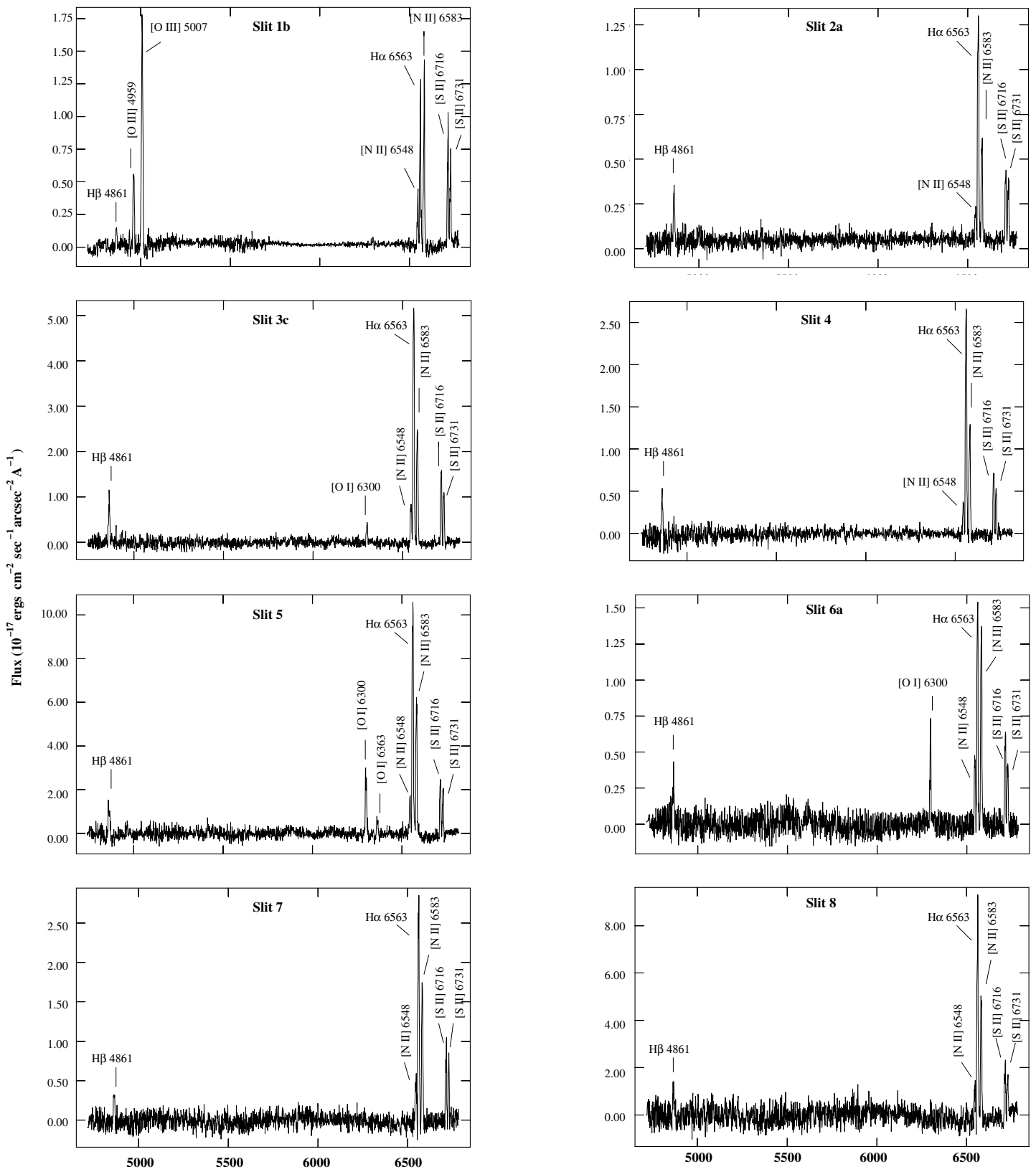

Wavelength( $(\AA)$

Fig. 3. Long-slit spectra from different positions of the observed area (see Table 1).

Table 3. New candidate SNRs.

\begin{tabular}{|c|c|c|c|c|c|c|}
\hline \multirow[t]{2}{*}{ SNR name } & \multicolumn{2}{|c|}{ SNR center } & \multirow{2}{*}{$\begin{array}{l}\text { Diameter } \\
\text { (arcmin) }\end{array}$} & \multicolumn{2}{|c|}{$N_{\mathrm{H}}^{a}$} & \multirow{2}{*}{$\begin{array}{c}N_{\mathrm{H}}^{b} \\
\mathrm{Ral}^{e} \& \mathrm{P} \& \mathrm{~S}^{f} \\
\left(\times 10^{21} \mathrm{~cm}^{-2}\right)\end{array}$} \\
\hline & $\begin{array}{c}\alpha \\
(\mathrm{h} \mathrm{m} \mathrm{s})\end{array}$ & $\begin{array}{c}\delta \\
\left({ }^{\circ}, \prime \prime\right)\end{array}$ & & $\begin{array}{c}\mathrm{Kal}^{c} \\
\left(\times 10^{21} \mathrm{~cm}^{-2}\right)\end{array}$ & $\begin{array}{c}\mathrm{D} \& \mathrm{~L}^{d} \\
\left(\times 10^{21} \mathrm{~cm}^{-2}\right)\end{array}$ & \\
\hline G 15.6-2.6 & 182846.1 & -163040 & $3.7 \times 10.2$ & 4.6 & 6.8 & $1.0-4.9$ \\
\hline G $15.8-2.8$ & 182935.8 & -162740 & $5.1 \times 12.9$ & 3.2 & 4.8 & $1.0-4.9$ \\
\hline G $15.8-2.2$ & 182738.8 & -161217 & $6.5 \times 8.7$ & 4.6 & 6.8 & $2.6-3.3$ \\
\hline G $15.8-1.9$ & 182630.7 & -160800 & $7.7 \times 8.3$ & 7.4 & 6.8 & $0.7-4.8$ \\
\hline G $16.2-2.5$ & 182923.5 & -160306 & $3.0 \times 7.2$ & 4.6 & 6.2 & $2.8-3.5$ \\
\hline G $15.6-2.7$ & 182853.5 & -163534 & $1.1 \times 1.1$ & 4.6 & 6.8 & $2.0-4.8$ \\
\hline
\end{tabular}

Notes. ${ }^{(a)} N_{\mathrm{H}}$ derived by the statistical relations in the direction of the candidate SNRs. ${ }^{(b)} N_{\mathrm{H}}$ calculated using current observations (min and $\max E(B-V)$ taken from Table 4) and the statistical relations. ${ }^{(c)}$ Kalberla et al. (2005) ; ${ }^{(d)}$ Dickey \& Lockman $(1990)$; ${ }^{(e)}$ Ryter et al. (1975) ; (f) Predehl \& Schmitt (1995) . 
spectra (slits $6 \& 7$ ), which is between 0.45 and 0.7 . There is also a bright filament further to the east (from $\alpha \simeq 18^{\mathrm{h}} 28^{\mathrm{m}} 28^{\mathrm{s}}$, $\delta \simeq-16^{\circ} 14^{\prime} 00^{\prime \prime}$ to $\left.\alpha \simeq 18^{\mathrm{h}} 28^{\mathrm{m}} 56^{\mathrm{s}}, \delta \simeq-16^{\circ} 15^{\prime} 05^{\prime \prime}\right)$ that displays shock-heated emission, but has however the weakest [S II] measured in the area $\left(\left[\mathrm{S}_{\mathrm{II}}\right] / \mathrm{ha}=0.45\right.$; slit 5$)$. According to its morphology, it is probable that it is unrelated to this remnant, although their correlation cannot be completely ruled out. There are also cases of radio and infrared emission that might correlate with those in the optical in some of the SNR's regions (Sect. 3.3), while [O III] emission has not been detected.

G 15.8-1.9. In contrast to the previous SNR candidates, this is quite faint in both the $\mathrm{H} \alpha+[\mathrm{N} \mathrm{II}]$ and $[\mathrm{S} \mathrm{II}]$ images, having less filamentary and more diffuse emission, while its [O III] emission was not detected. Its brightest part is aligned along the northnorthwest direction (from $\alpha \simeq 18^{\mathrm{h}} 26^{\mathrm{m}} 00^{\mathrm{s}}, \delta \simeq-16^{\circ} 04^{\prime} 25^{\prime \prime}$ to $\left.\alpha \simeq 18^{\mathrm{h}} 26^{\mathrm{m}} 40^{\mathrm{s}}, \delta \simeq-16^{\circ} 00^{\prime} 20^{\prime \prime}\right)$, there is fainter emission to the east ( $\left.\alpha \simeq 18^{\mathrm{h}} 26^{\mathrm{m}} 50^{\mathrm{s}}, \delta \simeq-16^{\circ} 13^{\prime} 45^{\prime \prime}\right)$, and no emission was detected to the south. The $\left[\mathrm{S}_{\mathrm{II}}\right] / \mathrm{H} \alpha$ ratios of 0.48 (spectrum, slit 8) and 0.50 (imaging) suggest that a shock-heated mechanism produced the emission, while the optical emission correlates well with the radio and partially with the infrared (Sect. 3.3).

G 16.2-2.5. This is the brightest candidate in $\mathrm{H} \alpha+\left[\mathrm{N}_{\mathrm{II}}\right]$ and $\left[\mathrm{S}_{\mathrm{II}}\right]$ with $\left[\mathrm{S}_{\mathrm{II}}\right] / \mathrm{H} \alpha \sim 0.5$. There are bright thick filaments around almost all the remnant (south from $\alpha \simeq 18^{\mathrm{h}} 28^{\mathrm{m}} 45^{\mathrm{s}}$, $\delta \simeq-16^{\circ} 06^{\prime} 10^{\prime \prime}$ to $\alpha \simeq 18^{\mathrm{h}} 29^{\mathrm{m}} 55^{\mathrm{s}}, \delta \simeq-16^{\circ} 03^{\prime} 30^{\prime \prime}$; west from $\alpha \simeq 18^{\mathrm{h}} 28^{\mathrm{m}} 45^{\mathrm{s}}, \delta \simeq-16^{\circ} 06^{\prime} 40^{\prime \prime}$ to $\alpha \simeq 18^{\mathrm{h}} 28^{\mathrm{m}} 56^{\mathrm{s}}, \delta \simeq$ $-16^{\circ} 01^{\prime} 30^{\prime \prime}$ and north-west to $\left.\alpha \simeq 18^{\mathrm{h}} 29^{\mathrm{m}} 14^{\mathrm{s}}, \delta \simeq-15^{\circ} 59^{\prime} 45^{\prime \prime}\right)$ with a width of $\sim 2-3^{\prime}$ (owing to the position of this candidate SNR, the north part can only be seen in Fig. 5). Weaker and more diffuse emission patches are located in its north-east area, while no [O III] emission was found. The detected emission-line structures are also bounded in all directions by diffuse emission, which is probably due to $\mathrm{H}_{\text {II }}$ regions. It is very close to the only known radio SNR G 16.2-2.7 but their position and morphology suggest that they are unrelated to each other (see Fig. 5). However, a small possibility that this emission is related to the radio remnant still remains if we consider that they can be seen at a different angle to the line of sight. Kinematic observations could either confirm or reject the latter scenario. There is also infrared emission in the area that partially correlates with the optical (Sect. 3.3).

G 15.6-2.7. This is the most peculiar region since it is the only area that shows strong [O III] and very strong [ $\left.\mathrm{N}_{\mathrm{II}}\right]$ emission. A shock-heated mechanism is responsible for the emission found since $[\mathrm{S}$ II] $/ \mathrm{H} \alpha>1.0$. Its morphology, size, and position in the diagnostic diagram (Fig. 4) enable us conclude that it might be a SNR that is unrelated to G 15.6-2.6, although their correlation cannot be ruled out so further investigation (i.e. highresolution spectroscopic observations) is needed to identify its origin. If they are related, then the possibility that its [S II] emission is background emission from G 15.6-2.6 and does not come from the object itself, will change the responsible mechanism to photoionization, and the object might then not be an SNR but a planetary nebula. We note that it is not unusual to find strong [O III] emission in both SNRs and PNe so it cannot be used as a criterion to help us distinguish between them. In Table 2, a typical [O III] flux is given. Radio emission is not found to be correlated with this SNR candidate, while there is faint infrared emission in its vicinity that might be associated with it (Sect. 3.3).

\subsection{The optical spectra}

Deep long-slit spectra were taken to accurately determine the nature of the observed emission by measuring the strengths of the $\mathrm{H} \alpha$ and $\left[\mathrm{S}_{\mathrm{II}}\right]$ emission lines. Eight different spectra, extracted from the relatively brightest optical filaments, are shown in Fig. 3 and the measured fluxes are given in Table 4. Different apertures $(\mathrm{a}, \mathrm{b}$, and $\mathrm{c})$ were extracted from each spectrum along the slit that had an offset (see Table 1) either north or south of the slit center. The selection criteria for the extraction apertures were to be in an area free of field stars including sufficient line emission to permit an accurate determination of the observed line fluxes. The background extraction aperture was taken towards either the northern or southern end of the slits depending on the slit position. The $\mathrm{S} / \mathrm{N}$ 's presented in Table 4 do not include calibration errors, which are smaller than 10 percent.

The properties of the spectra point strongly to emission from shock-heated gas $([\mathrm{S}$ II $] / \mathrm{H} \alpha$ ratios between 0.44 and 1.20, $[\mathrm{N}$ II $] / \mathrm{H} \alpha$ ratios between 0.53 and 1.35 , and the presence of [O I] emission; see Table 4). The filamentary nature of the newly discovered optical radiation, as seen in the narrow-band images, also supports this conclusion. The absolute $\mathrm{H} \alpha$ flux covers a range of values from 0.6 to $41.3 \times 10^{-17} \mathrm{erg} \mathrm{s}^{-1} \mathrm{~cm}^{-2} \operatorname{arcsec}^{-2}$. The $[\mathrm{S}$ II] $] \lambda \lambda 6716 / 6731$ ratio, which was measured to be between 1.2 and 1.5, indicates low electron densities (below $240 \mathrm{~cm}^{-3}$ based on Osterbrock \& Ferland 2006, and below $400 \mathrm{~cm}^{-3}$ by taking into account the statistical errors in the sulfur lines; Shaw \& Dufour 1995).

Although, the low ionization lines are quite strong, [O III] line emission at $5007 \AA$ is only detected at position 1 (Table 2), suggesting that there are shock velocities $\sim 120 \mathrm{~km} \mathrm{~s}^{-1}$ (Cox \& Raymond 1985) and below $200 \mathrm{~km} \mathrm{~s}^{-1}$ (Allen et al. 2008 ) in that area. In addition, the $[\mathrm{O} \mathrm{III}] / \mathrm{H} \beta$ is $>6$, according to theoretical models (Raymond et al. 1988), which is indicative of shocks with incomplete recombination zones. The relatively weak $\mathrm{H} \beta$ emission and the absence of [O III] emission in all the other positions suggest that there is significant interstellar attenuation of the optical emission that could be explained by slow shocks propagating into the interstellar clouds $\left(\leq 100 \mathrm{~km} \mathrm{~s}^{-1}\right.$; Hartigan et al. 1987).

The $\log \left(\mathrm{H} \alpha /\left[\mathrm{N}_{\mathrm{II}}\right]\right)$ versus $\log \left(\mathrm{H} \alpha /\left[\mathrm{S}_{\mathrm{II}}\right]\right)$ intensities, from Table 4, corrected for interstellar extinction, are compared with others of well-defined phenomena in Fig. 4 (following Sabbadin et al. 1977; and Cantó 1981) and show that all positions are either within or very close to (taking into account the calculated error for those that are not within) the area designated for SNRs.

\subsection{Observations at other wavelengths}

Radio emission in the area of the optical structures is detected in the low-resolution ( $7^{\prime}$ ) $4850 \mathrm{MHz}$ images of the Green Bank survey (Condon et al. 1991). In most cases, the radio emission matches the optical emission, but given the low resolution it is hard to establish a detailed spatial correlation, apart from confirming their association. We also examined the higher resolution CGPS data at $1420 \mathrm{MHz}$ (Taylor et al. 2003), but no prominent emission was found to be correlated with the optical. The observed filaments are located close to radio contours (Fig. 5), in many cases there is a good correlation but the low resolution of the radio images does not allow us to determine the position of the filament relative to the shock front. Higher-resolution radio observations at different wavelengths should be performed to provide conclusive evidence that the sources are SNRs. 
Table 4. Relative line fluxes.

\begin{tabular}{|c|c|c|c|c|c|c|c|c|c|c|c|c|c|c|c|}
\hline \multirow[b]{2}{*}{ Line $(\AA)$} & \multicolumn{3}{|c|}{ Slit 1a } & \multicolumn{3}{|c|}{ Slit $1 b$} & \multicolumn{3}{|c|}{ Slit 2a } & \multicolumn{3}{|c|}{ Slit $2 b$} & \multicolumn{3}{|c|}{ Slit 2c } \\
\hline & $F^{a}$ & $I^{b}$ & $S / N^{c}$ & $F$ & $I$ & $S / N$ & $F$ & $I$ & $S / N$ & $F$ & $I$ & $S / N$ & $F$ & $I$ & $S / N$ \\
\hline $\mathrm{H} \beta 4861$ & 23 & 35 & 13 & 15 & 35 & 2 & 19 & 35 & 27 & 21 & 35 & 18 & 24 & 35 & 21 \\
\hline [O III] 4959 & 13 & 20 & 7 & 66 & 147 & 5 & - & - & - & - & - & - & - & - & - \\
\hline [O III] 5007 & 36 & 53 & 23 & 187 & 405 & 13 & - & - & - & - & - & - & - & - & - \\
\hline [OI] 6300 & 14 & 14 & 17 & - & - & - & - & - & - & - & - & - & - & - & - \\
\hline$\left[\mathrm{N}_{\text {II }}\right] 6548$ & 32 & 32 & 36 & 36 & 36 & 5 & 14 & 14 & 42 & 15 & 15 & 22 & 13 & 12 & 42 \\
\hline $\mathrm{H} \alpha 6563$ & 100 & 100 & 89 & 100 & 100 & 14 & 100 & 100 & 216 & 100 & 100 & 123 & 100 & 100 & 137 \\
\hline [N II] 6584 & 95 & 95 & 82 & 100 & 99 & 14 & 40 & 39 & 103 & 44 & 43 & 58 & 42 & 42 & 66 \\
\hline [S II] 6716 & 63 & 61 & 59 & 74 & 69 & 10 & 27 & 26 & 71 & 31 & 29 & 44 & 30 & 29 & 44 \\
\hline [S II] 6731 & 49 & 47 & 47 & 53 & 49 & 7 & 22 & 21 & 56 & 26 & 25 & 38 & 26 & 23 & 38 \\
\hline Absolute $\mathrm{H} \alpha$ flux $^{d}$ & \multicolumn{3}{|c|}{7.5} & \multicolumn{3}{|c|}{0.62} & \multicolumn{3}{|c|}{16.7} & \multicolumn{3}{|c|}{13.3} & \multicolumn{3}{|c|}{11.9} \\
\hline$[\mathrm{S}$ II $] / \mathrm{H} \alpha$ & \multicolumn{3}{|c|}{$1.08 \pm 0.02$} & \multicolumn{3}{|c|}{$1.20 \pm 0.1$} & \multicolumn{3}{|c|}{$0.47 \pm 0.03$} & \multicolumn{3}{|c|}{$0.54 \pm 0.05$} & \multicolumn{3}{|c|}{$0.51 \pm 0.04$} \\
\hline$F(6716) / F(6731)$ & \multicolumn{3}{|c|}{$1.30 \pm 0.04$} & & $.4 \pm 0$ & & & $1.3 \pm$ & & & $1.2 \pm 0$ & & & $1.3 \pm 0$ & \\
\hline$[\mathrm{N}$ II $] / \mathrm{H} \alpha$ & & $.28 \pm$ & 02 & & $35 \pm$ & & & $53 \pm$ & & & $.58 \pm 0$ & & & $54 \pm 0$ & \\
\hline $\mathrm{c}(\mathrm{H} \beta)^{e}$ & & $.52 \pm$ & 10 & & $.1 \pm 0$ & & & $74 \pm$ & & & $64 \pm 0$ & & & $50 \pm 0$ & \\
\hline$E_{B-V}$ & & $.37 \pm($ & .07 & & $.7 \pm 0$ & & & $51 \pm$ & & & $44 \pm 0$ & & & $34 \pm 0$ & \\
\hline & & Slit 3 & & & Slit 3 & & & Slit 3 & & & Slit 4 & & & Slit 5 & \\
\hline Line $(\AA)$ & $F$ & $I$ & $S / N$ & $F$ & $I$ & $S / N$ & $F$ & $I$ & $S / N$ & $F$ & $I$ & $S / N$ & $F$ & $I$ & $S / N$ \\
\hline $\mathrm{H} \beta 4861$ & 15 & 35 & 18 & 20 & 35 & 44 & 18 & 35 & 32 & 28 & 35 & 16 & 20 & 35 & 21 \\
\hline [O I $] 6300$ & 9 & 10 & 26 & 5 & 5 & 33 & 7 & 7 & 31 & - & - & - & 24 & 25 & 61 \\
\hline [O I ] 6363 & - & - & - & - & - & - & - & - & - & - & - & - & 12 & 12 & 27 \\
\hline$[\mathrm{N}$ II $] 6548$ & 17 & 16 & 38 & 16 & 16 & 85 & 18 & 18 & 66 & 15 & 14 & 21 & 15 & 15 & 37 \\
\hline $\mathrm{H} \alpha 6563$ & 100 & 100 & 165 & 100 & 100 & 337 & 100 & 100 & 243 & 100 & 100 & 115 & 100 & 100 & 194 \\
\hline$[\mathrm{N}$ II $] 6584$ & 96 & 95 & 73 & 44 & 44 & 180 & 49 & 48 & 141 & 47 & 47 & 58 & 57 & 56 & 122 \\
\hline [S II] 6716 & 63 & 60 & 28 & 28 & 26 & 115 & 31 & 29 & 94 & 27 & 26 & 36 & 27 & 26 & 57 \\
\hline [S II] 6731 & 49 & 45 & 16 & 20 & 18 & 88 & 21 & 19 & 70 & 21 & 20 & 28 & 20 & 19 & 42 \\
\hline Absolute $\mathrm{H} \alpha$ flux & & 14.1 & & & 27.0 & & & 26.3 & & & 10.7 & & & 20.2 & \\
\hline$[\mathrm{S}$ II $] / \mathrm{H} \alpha$ & & $.05 \pm$ & .09 & & $45 \pm 0$ & & & $49 \pm$ & & & $47 \pm 0$ & & & $45 \pm 0$ & \\
\hline$F(6716) / F(6731)$ & & $1.3 \pm$ & & & $41 \pm 0$ & & & $1.5 \pm$ & & & $1.3 \pm 0$ & & & $1.4 \pm 0$ & \\
\hline$\left[\mathrm{N}_{\mathrm{II}}\right] / \mathrm{H} \alpha$ & & $1.1 \pm($ & & & $60 \pm 0$ & & & $66 \pm$ & & & $61 \pm 0$ & & & $71 \pm 0$ & \\
\hline$c(\mathrm{H} \beta)$ & & $.04 \pm$ & 07 & & $70 \pm 0$ & & & $82 \pm$ & & & $27 \pm 0$ & & & $69 \pm 0$ & \\
\hline$E_{B-V}$ & & $.57 \pm$ & & & $19 \pm 0$ & & & $72 \pm$ & & & $48 \pm 0$ & & & $48 \pm 0$ & \\
\hline & & Slit 6 & & & Slit 6 & & & Slit & & & Slit 8 & & & & \\
\hline Line $(\AA)$ & $F$ & $I$ & $S / N$ & $F$ & $I$ & $S / N$ & $F$ & $I$ & $S / N$ & $F$ & $I$ & $S / N$ & & & \\
\hline $\mathrm{H} \beta 4861$ & 27 & 35 & 13 & 30 & 35 & 24 & 16 & 35 & 8 & 19 & 35 & 10 & & & \\
\hline [O I $] 6300$ & 31 & 31 & 35 & 21 & 21 & 40 & - & - & - & - & - & - & & & \\
\hline$\left[\mathrm{N}_{\text {III }}\right] 6548$ & 37 & 37 & 36 & 20 & 21 & 39 & 23 & 23 & 22 & 16 & 16 & 23 & & & \\
\hline $\mathrm{H} \alpha 6563$ & 100 & 100 & 84 & 100 & 100 & 146 & 100 & 100 & 81 & 100 & 100 & 111 & & & \\
\hline$\left[\mathrm{N}_{\text {II }}\right] 6584$ & 95 & 94 & 73 & 66 & 65 & 96 & 67 & 66 & 55 & 62 & 61 & 71 & & & \\
\hline [S II] 6716 & 45 & 44 & 39 & 26 & 25 & 41 & 31 & 29 & 27 & 29 & 28 & 38 & & & \\
\hline [S II] 6731 & 30 & 29 & 27 & 19 & 19 & 30 & 21 & 19 & 20 & 21 & 20 & 30 & & & \\
\hline Absolute $\mathrm{H} \alpha$ flux & & 8.3 & & & 13.0 & & & 19.7 & & & 41.3 & & & & \\
\hline$[\mathrm{S}$ II $] / \mathrm{H} \alpha$ & & $0.7 \pm$ & & & $44 \pm 0$ & & & $0.5 \pm$ & & & $48 \pm 0$ & & & & \\
\hline$F(6716) / F(6731)$ & & $1.5 \pm$ & & & $.3 \pm 0$ & & & $1.5 \pm$ & & & $1.4 \pm 0$ & & & & \\
\hline$\left[\mathrm{N}_{\mathrm{II}}\right] / \mathrm{H} \alpha$ & & $1.3 \pm$ & & & $86 \pm 0$ & & & $0.9 \pm$ & & & $0.8 \pm 0$ & & & & \\
\hline$c(\mathrm{H} \beta)$ & & $.34 \pm$ & & & $20 \pm 0$ & & & $1.0 \pm$ & & & $0.7 \pm 0$ & & & & \\
\hline$E_{B-V}$ & & $.23 \pm($ & .07 & & $13 \pm 0$ & & & $0.7 \pm$ & & & $.51 \pm 0$ & & & & \\
\hline
\end{tabular}

Notes. The errors in the emission line ratios are calculated through standard error propagation. ${ }^{(a)}$ Observed fluxes normalized to $F(\mathrm{H} \alpha)=100$ and uncorrected for interstellar extinction. ${ }^{(b)}$ Intrinsic surface brightness normalized to $I(\mathrm{H} \alpha)=100$ and corrected for interstellar extinction. (c) Numbers represent the $\mathrm{S} / \mathrm{N}$ ratio of the quoted fluxes. (d) In units of $10^{-17} \mathrm{erg} \mathrm{s}^{-1} \mathrm{~cm}^{-2} \operatorname{arcsec}^{-2}$. (e) The logarithmic extinction is derived by $c=1 / 0.348 \times \log \left((\mathrm{H} \alpha / \mathrm{H} \beta)_{\mathrm{obs}} / 2.85\right)$.

We have also searched ROSAT All-sky survey data for X-ray emission, but it is very faint and diffuse and cannot identify the sources in detail. We also searched the VLA Galactic Plane Survey (VGPS; Stil et al. 2006) for H i kinematical data but unfortunately the area of interest was not covered by this survey.

To explore how the optical emission correlates with the infrared, we searched all the available data at NASA/IPAC Infrared Science Archive. In particular, we examined the GLIMPSE/Spitzer (Churchwell et al. 2009), the WISE (Wright et al. 2010), and the IRIS (Miville-Deschênes \& Lagache 2005) images of the same area. Unfortunately, the area in which we are interested was not covered by the GLIMPSE survey at 3.6, $4.5,5.8$, and $8.0 \mu \mathrm{m}$ (resolution at $1.2^{\prime \prime}$ ), while the IRIS data at 25,60 , and $100 \mu \mathrm{m}$ (resolution from $30^{\prime \prime}$ to $2^{\prime}$ ) revealed a clear enhancement of infrared emission in the area where the optical emission of the candidate SNRs is detected, although the lowresolution maps do not permit us to make a detailed comparison. On the other hand, the WISE data at 3.4, 4.6, 12, and $22 \mu \mathrm{m}$ (with resolutions of 6.1, 6.4, 6.5, and 12", respectively) show evidence of a correlation with the optical and/or radio emission 


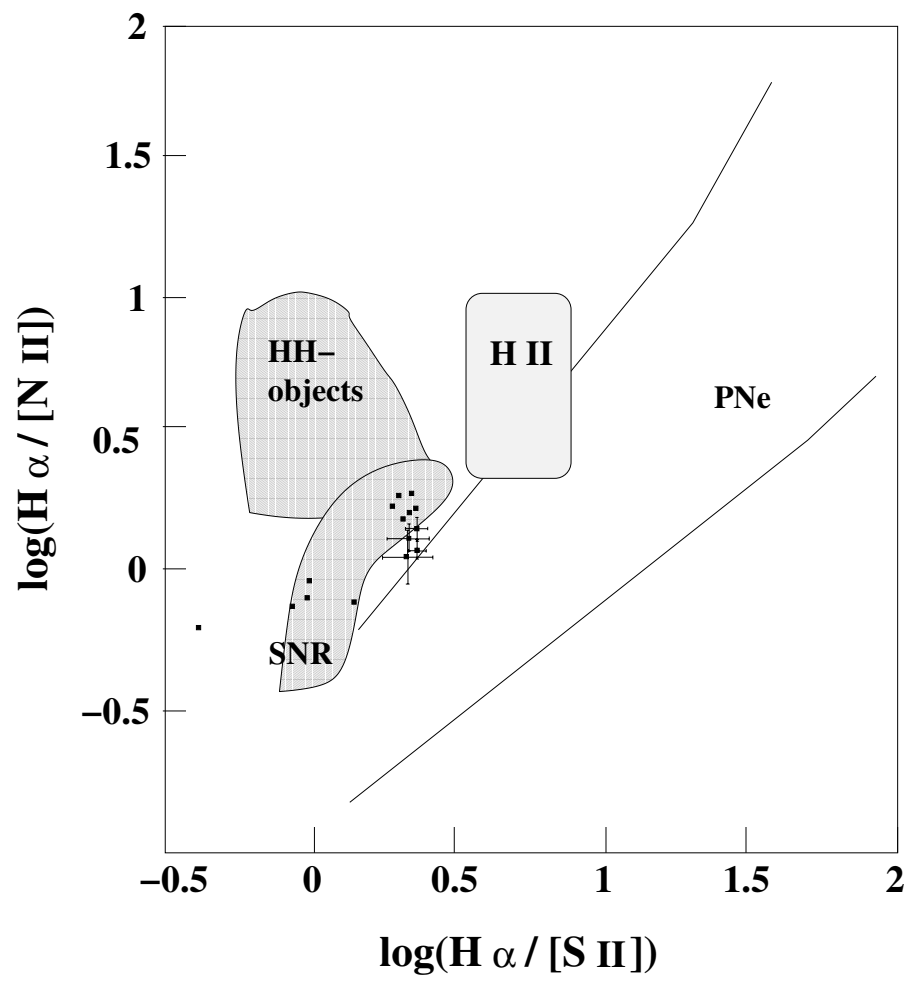

Fig. 4. Diagnostic diagram (Sabbadin et al. 1977; Cantó 1981), where the positions of line ratios listed in Table 1, from slit 1a to 8, are shown as black squares. For positions that are not within the SNR region, the calculated errors have taken into account.

in the last two channels. In particular, both the $12 \mu \mathrm{m}$ and $22 \mu \mathrm{m}$ images show evidence of associations with the optical and radio filamentary structures of the candidate SNRs. Since the $12 \mu \mathrm{m}$ image has essentially the same features as the $22 \mu \mathrm{m}$ image but that appear to be stronger in the latter, in Fig. 6 we present a grayscale representation of the $22 \mu \mathrm{m}$ image with the overlapping contours of the optical emission. In a few cases, the infrared emission seems to follow the morphology of the candidate SNRs suggesting that they are possibly associated, which is expected since it is known that the shocked gas cools through emission lines, and that many important emission lines occur in the midinfrared. A similar case is that of the SNR G 296.7-0.9 (Robbins et al. 2012) where a possible association of this SNR with the infrared emission was found. It should also be noted that the appearance of the infrared emission in the vicinity and not on the optical emission in most of the cases is expected, since in general there is not a very good correlation between the infrared and H $\alpha$ emission (Reach et al. 2006).

\section{Discussion}

Our newly discovered candidate SNRs towards the Sagittarius constellation show up as incomplete circular or elliptical structures in the optical and in most cases in the radio, but have no $\mathrm{X}$-ray and $\mathrm{H}$ I emission detections so far. The absence of soft $\mathrm{X}$-ray emission may indicate a low shock temperature and/or a low density of the local ISM. Its optical emission marginally correlates with the infrared. The elliptical shapes of the candidate SNRs are unusual compared to those of most known SNRs, suggesting that the surrounding medium is very irregular and of variable interstellar density. However, it should be mentioned that a significant number of known SNRs do not have circular structure (but instead bilateral, barriel, elliptical, cylindrical etc.) depending on their position relative to the line of sight so this might also be a reason for their appearance.

Detailed optical observations have been performed in an attempt to understand the nature of the candidate SNRs. The lower ionization images in $\mathrm{H} \alpha+\left[\mathrm{N}_{\mathrm{II}}\right]$ and [S II] reveal several filamentary and diffuse structures, while the higher ionization image in $[\mathrm{O}$ III] only shows emission in one region. The $\mathrm{H} \alpha+[\mathrm{N}$ II] image most clearly describes the newly detected structures. Sulfur line emission is also detected and generally appears to be less filamentary and more diffuse than in the $\mathrm{H} \alpha+\left[\mathrm{N}_{\mathrm{II}}\right]$ image with their position and shape agreeing with those in the $\mathrm{H} \alpha+[\mathrm{N}$ II] image. The [O III] flux production depends mainly on the shock velocity and the ionization state of the preshocked gas. Therefore, as mentioned in Sect. 3.1, the absence of [O III] emission in almost all areas may be explained by slow shocks propagating into the ISM. The presence of [O I ] $6300 \AA$ line emission is also consistent with the emission being from shocked material. The $[\mathrm{O} \mathrm{III}] / \mathrm{H} \beta$ ratio is a very useful diagnostic tool for complete or incomplete shock structures (Raymond et al. 1988). However, the absence of [O III] does not allow us to suggest that there are either complete or incomplete shock structures apart from position 1 where shocks with incomplete recombination zones should be present. Both the calibrated images and the long-slit spectra suggest that the detected emission results from shock-heated gas since the $\left[\mathrm{S}_{\mathrm{II}}\right] / \mathrm{H} \alpha$ ratio exceeds the empirical SNR criterion value of $0.4-0.5$, while the measured $[\mathrm{N} \mathrm{II}] / \mathrm{H} \alpha$ ratio also confirms this result.

The SNR origin of the proposed candidate remnants is strongly suggested by the values of the line ratios in Fig. 4 compared with those of Herbig-Haro objects, $\mathrm{H}$ in regions, and planetary nebulae (PNe). The observed SNRs closely follow the trend of known shock-ionized, evolved SNRs.

The $\mathrm{H} \alpha / \mathrm{H} \beta$ ratios in Table 4 can be used to estimate the variations in the logarithmic extinction coefficient $\mathrm{c}$ over these sources, assuming an intrinsic ratio of 3 and the interstellar extinction curve implemented in the nebular package (Shaw \& Dufour 1995) within the IRAF software. An interstellar extinction c (see Table 4) between 0.2 and 1.1 or an $A_{\mathrm{V}}$ between 0.4 and 2.2 were measured, respectively. We also determined the electron density by measuring the density-sensitive line ratio of [S II] $\lambda \lambda$ 6716/6731. The measured densities lie below $240 \mathrm{~cm}^{-3}$.

The candidate remnants under investigation have not been studied in the past, hence the current stage of their evolution is unknown. Our aim is to provide a first indication of their stage of evolution by estimating basic SNR parameters, assuming that the temperature is close to $10^{4} \mathrm{~K}$.

Estimated values of $N_{\mathrm{H}}$ between 4.8 and $6.8 \times 10^{21} \mathrm{~cm}^{-2}$ and $N_{\mathrm{H}}$ between 3.2 and $7.4 \times 10^{21} \mathrm{~cm}^{-2}$ are given by Dickey \& Lockman (1990) and Kalberla et al. (2005) respectively, for the column density in the direction of the candidate remnants. Using the relations of Ryter et al. (1975) and Predehl \& Schmitt (1995), we obtain $N_{\mathrm{H}}$ between 0.9 and $4.9 \times 10^{21} \mathrm{~cm}^{-2}$ for the minimum and maximum $\mathrm{c}$ values calculated from our spectra. In Table 3, we present the estimated values of $N_{\mathrm{H}}$ for each candidate remnant, where it can be seen that the values based on the optical data and the statistical relations are consistent with the estimated Galactic $N_{\mathrm{H}}$ from Kalberla et al. (2005) and less than that estimated from Dickey \& Lockman (1990). However, it should be noted that the slightly higher values calculated by the latter method can be explained by its additional coverage of gas beyond the area of interest. Assuming that they are still in the adiabatic phase of their evolution, the preshock cloud 
DEC

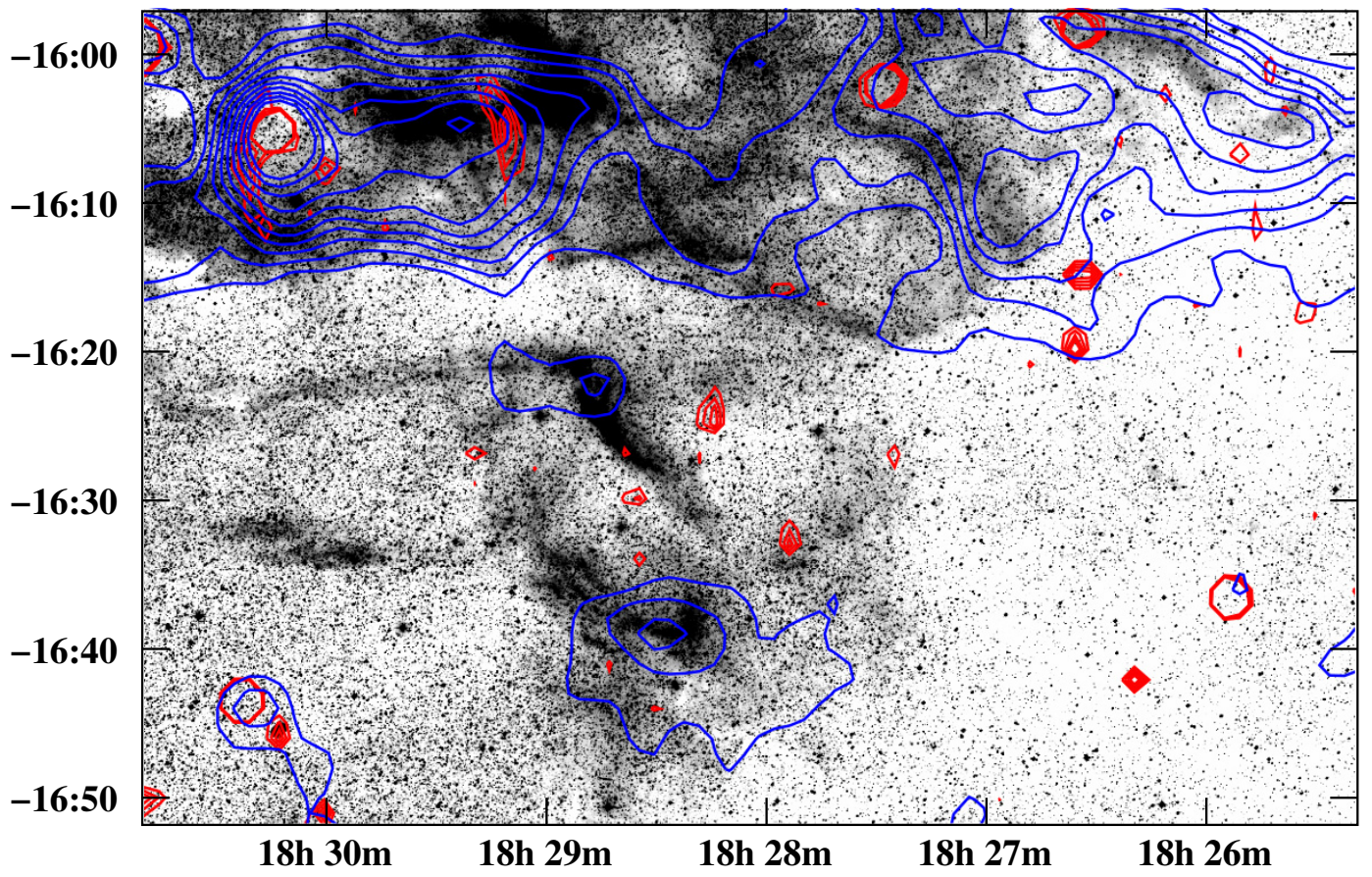

\section{R.A.}

Fig. 5. The correlation between the SuperCOSMOS $\mathrm{H} \alpha$ mosaic image and the radio emission from archival data at $4850 \mathrm{MHz}$ (blue line) and $1400 \mathrm{MHz}$ (red line). The blue contours scale linearly from $2.0 \times 10^{-2} \mathrm{Jy} /$ beam to $0.2 \mathrm{Jy} /$ beam, with steps of $0.02 \mathrm{Jy} / \mathrm{beam}$ and the red from $1.2 \times 10^{-3} \mathrm{Jy} /$ beam to $4.0 \times 10^{-3} \mathrm{Jy} /$ beam, with steps of $9.3 \times 10^{-4} \mathrm{Jy} / \mathrm{beam}$.

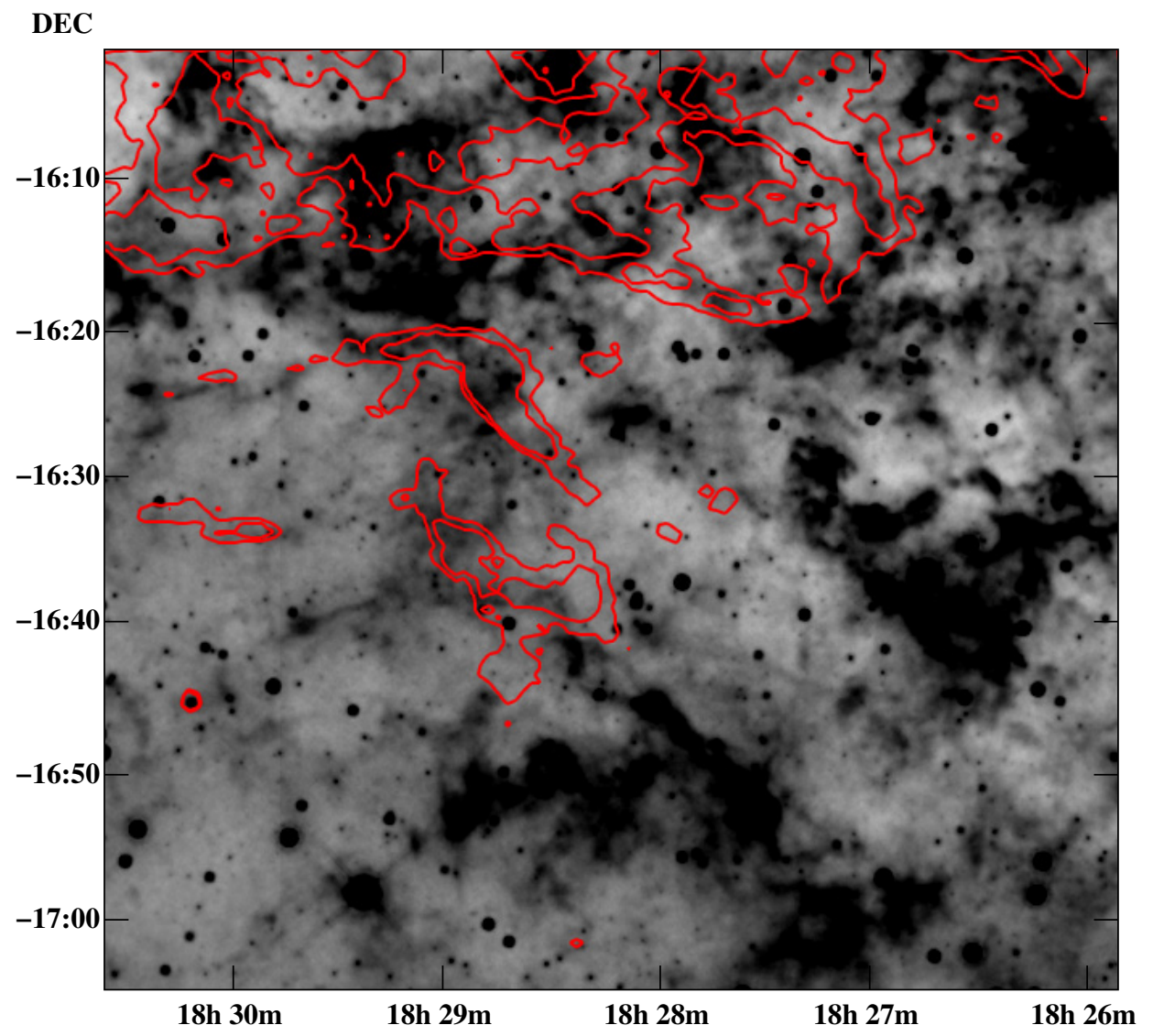

R. A.

Fig. 6. The correlation between the infrared WISE emission at $22 \mu \mathrm{m}$ image and the $\mathrm{H} \alpha+[\mathrm{N}$ II] emission (red lines). The red contours values are 70 (low) and 130 (high) $\times 10^{-17} \mathrm{erg} \mathrm{s}^{-1} \mathrm{~cm}^{-2} \operatorname{arcsec}^{-2}$. 
Table 5. Pulsars found within a region of $1.5^{\circ}$ from the candidate SNRs.

\begin{tabular}{cccccc}
\hline \hline Pulsar name & \multicolumn{2}{c}{ Pulsar center } & Period & Candidate SNR $^{a}$ & References \\
& $\begin{array}{c}\alpha \\
(\mathrm{h} \mathrm{m} \mathrm{s})\end{array}$ & $\left({ }^{\circ}{ }^{\prime \prime \prime}\right)$ & $(\mathrm{s})$ & & \\
\hline $\mathrm{J} 1822-1606$ & 182223.0 & -160559.0 & 8.4377 & 3,4 & $(1),(2)$ \\
$\mathrm{J} 1822-1617$ & 182236.6 & -161735.0 & 0.8311 & 3,4 & $(3)$ \\
$\mathrm{J} 1823-1526$ & 182321.4 & -152622.0 & 1.6254 & 3,4 & $(3)$ \\
$\mathrm{J} 1824-1500$ & 182414.1 & -150033.0 & 0.4122 & 3,4 & $(3)$ \\
$\mathrm{J} 1825-1446$ & 182502.9 & -144652.6 & 0.2792 & 4 & $(4),(5)$ \\
$\mathrm{J} 1826-1526$ & 182612.6 & -152603.0 & 0.3820 & $1-6$ & $(6)$ \\
$\mathrm{J} 1829-1751$ & 182943.1 & -175103.9 & 0.3071 & $1,2,6$ & $(5),(7),(8)$ \\
$\mathrm{J} 1834-1710$ & 183453.4 & -175103.9 & 0.3583 & 2 & $(9)$ \\
\hline
\end{tabular}

Notes. ${ }^{(a)}$ Candidate SNRs numbered according to their order presented in Table 3.

References. (1) Rea et al. (2011); (2) Cummings et al. (2011); (3) Hobbs et al. (2004a); (4) Clifton \& Lyne (1986); (5) Hobbs et al. (2004b); (6) Morris et al. (2002); (7) Davies et al. (1972); (8) Zou et al. (2005); (9) Kramer et al. (2003).

density $n_{\mathrm{c}}$ can be measured by using the relationship (Dopita 1979)

$n_{[\mathrm{SII}]} \simeq 45 n_{\mathrm{c}} V_{\mathrm{s}}^{2} \mathrm{~cm}^{-3}$,

where $n_{[\mathrm{SII}]}$ is the electron density derived from the sulfur line ratio and $V_{\mathrm{s}}$ is the shock velocity in the clouds in units of $100 \mathrm{~km} \mathrm{~s}^{-1}$. Furthermore, the blast wave energy can be expressed in terms of the cloud parameters by using the equation given by McKee \& Cowie (1975)

$E_{51}=2 \times 10^{-5} \beta^{-1} n_{\mathrm{c}} V_{\mathrm{s}}^{2} r_{\mathrm{s}}^{3}$ erg.

The factor $\beta$ is approximately equal to 1 at the blast-wave shock, $E_{51}$ is the explosion energy in units of $10^{51} \mathrm{erg}$, and $r_{\mathrm{s}}$ is the radius of the remnant in pc.

By using the upper limit to the electron density of $240 \mathrm{~cm}^{-3}$, which was derived from our spectra, we obtain from Eq. (1) that $n_{\mathrm{c}} V_{\mathrm{s}}^{2}<5.3$. Equation (2) then becomes $E_{51}<\alpha \times 10^{-3} D_{1 \mathrm{kpc}}^{3}$, where $\alpha$ is a value that depends on the diameter of each candidate remnant and $D_{1 \mathrm{kpc}}$ is the distance to the remnant in units of $1 \mathrm{kpc}$. For the different diameters of the remnants and assuming a typical value of 1 for the supernova explosion energy $\left(E_{51}\right)$, we derive that the candidate SNRs lie at distances greater than $8 \mathrm{kpc}$. Since there are no other measurements of the interstellar density $n_{0}$, values of 0.1 and 1.0 will be examined. The lower interstellar density of $\sim 0.1 \mathrm{~cm}^{-3}$ then suggests that their distance is between $10 \mathrm{kpc}$ and $22 \mathrm{kpc}$, while for $n_{0} \approx 1 \mathrm{~cm}^{-3}$ it is between 1.0 and $2.2 \mathrm{kpc}$ for the lower and higher column densities calculated above. Combining the previous results, values between $0.1 \mathrm{~cm}^{-3}$ and $0.2 \mathrm{~cm}^{-3}$ for the interstellar density seem to be more probable. We note that the ambient density of gas around the candidate SNRs is not the same so this might also change the estimated values.

We also searched for pulsars in the region using the ATNF Pulsar Catalogue (Manchester et al. 2005). In total, we found 8 pulsars within a $1.5^{\circ}$ diameter circle away from the center of each candidate SNR. In Table 5, we present their names, coordinates, and rotation period as well as which candidate SNRs fulfill the $1.5^{\circ}$ limit. The closest one to G $15.8-1.9$ is PSR18261526 , at a distance of $0.7^{\circ}$. It is unclear at the moment whether any of these pulsars are related to the candidate SNRs, although the existence of a significant number of pulsars very close to the area of the candidate SNRs is another strong indication of more than one SNR in the region. It is more plausible that owing to their distance from the candidate SNRs, they are unrelated to them since the closest pulsar is about 4 radii from the nearest
SNR or $>75 \mathrm{pc}$ in the plane of the sky at a distance of $8 \mathrm{kpc}$. However, if they are not in the plane of the sky, which is probably the case, then these numbers might change. In addition, they might be at different distances which would also affect the numbers. Therefore, their correlation can be neither confirmed nor ruled out and should be examined in greater detail in the future.

It is possible that this irregular group of filaments is part of a wider structure but is being seen through holes in intervening clouds, leading to patchy optical interstellar-extinction. The current data are insufficient for us to claim such a strong a correlation. Furthermore, there is a possibility that the detected optical emission could be part of a number of supernova explosions in the area. However, since neither the distance nor the ISM density are accurately known, we cannot confidently determine the current stage of evolution of the candidate remnants and more observations are needed.

Further study of the area would benefit from higherresolution multiwavelength observations (optical, radio, and $\mathrm{X}$-ray) which would help us reduce the current uncertainties. In particular, higher-resolution imaging observations will help us to verify whether they have filamentary structures and confirm their morphological appearance, while kinematic observations will help us to determine their three-dimensional morphology and measure their expansion velocities. X-ray observations of the filaments will help us ascertain whether there is any correlation between the faint and diffuse X-ray emission and the optical filaments and provide more information about their evolutionary stage, while radio observations would also be useful to examine their non-thermal spectral index and confirm their SNR nature.

Acknowledgements. We thank the referee for his constructive comments and suggestions that helped to improve the manuscript significantly. Skinakas Observatory is a collaborative project of the University of Crete, the Foundation for Research and Technology-Hellas and the Max-Planck-Institut für Extraterrestrische Physik. This research made use of data from the SuperCOSMOS H $\alpha$ Survey (AAO/UKST), the ATNF Pulsar Catalogue, and the NASA/IPAC Infrared Science Archive.

\section{References}

Allen, M. G., Groves, B. A., Dopita, M. A., Sutherland, R. S., \& Kewley, L. J. 2008, ApJS, 178, 20

Boumis, P., Paleologou, E. V., Mavromatakis, F., \& Papamastorakis, J. 2003, MNRAS, 339, 735

Boumis, P., Akras, S., Xilouris, E. M., et al. 2006, MNRAS, 367, 1551

Boumis, P., Alikakos, J., Christopoulou, P. E., et al. 2008, A\&A, 481, 705 
J. Alikakos et al.: Discovery of optical candidate supernova remnants in Sagittarius

Boumis, P., Xilouris, E. M., Alikakos, J., et al. 2009, A\&A, 499, 789 Cantó, J. 1981, in Investigating the Universe (Dordrecht: Reidel), 95 Churchwell, E., Babler, B. L., Meade, M. R., et al. 2009, PASP, 121, 213

Clifton, T. R., \& Lyne, A. G. 1986, Nature, 320, 43

Condon, J. J., Broderick, J. J., \& Seielstad, G. A. 1991, AJ, 102, 2041

Cox, D. P., \& Raymond, J. C. 1985, ApJ, 298, 651

Cummings, J. R., Burrows, D., Campana, S., et al. 2011, ATel, 3488, 1 Davies, J., Lyne, A. G., \& Seiradakis, J. H. 1972, Nature, 240, 229

Dickey, J. M., \& Lockman, F. J. 1990, ARA\&A, 28, 215

Dopita, M. A. 1979, ApJS, 40, 455

Green, D. A. 2009, Bull. Astron. Soc. India, 37, 45

Hamuy, M., Walker, A. R., Suntzeff, N. B., et al. 1992, PASP, 104, 533

Hartigan, P., Raymond, J., \& Hartmann, L. 1987, ApJ, 316, 323

Hobbs, G., Faulkner, A., Stairs, I. H., et al. 2004a, MNRAS, 352, 1439

Hobbs, G., Lyne, A., Kramer, M., Martin, C. E., \& Jordan, C. 2004b, MNRAS, 353, 1311

Kalberla, P. M. W., Burton, W. B., Hartmann, D., et al. 2005, A\&A, 440, 775

Kramer, M., Bell, J. F., Manchester, R. N., et al. 2003, MNRAS, 342, 1299

Lasker, B. M., Lattanzi, M. G., McLean, B. J., et al. 2008, AJ, 136, 735

Manchester, R. N., Hobbs, G. B., Teoh, A., \& Hobbs, M. 2005, AJ, 129, 1993

Mannucci, F., Della Valle, M., Panagia, N., et al. 2005, A\&A, 433, 807

McKee, C. F., \& Cowie, L. L. 1975, ApJ, 195, 715
Miville-Deschênes, M-A., \& Lagache, G. 2005, ApJS, 157, 302

Morris, D. J., Hobbs, G., Lyne, A. G., et al. 2002, MNRAS, 335, 275

Osterbrock, D. E., \& Ferland, G. J. 2006, Astrophysics of gaseous nebulae and AGN (US: University Science Books)

Parker, Q. A., Phillipps, S., Pierce, M. J., et al. 2005, MNRAS, 362, 689

Predehl, P., \& Schmitt, J. H. M. M. 1995, A\&A, 293, 889

Raymond, J. C., Hester, J. J., Cox, D., et al. 1988, ApJ, 324, 869

Rea, N., Esposito, P., Israel, G. L., Tiengo, A., \& Zan, S. 2011, ATel, 3501, 1

Reach, W. T., Rho, J., Tappe, A., et al. 2006, AJ, 131, 1479

Reynolds, S. P., Borkowski, K. J., Green, D. A., et al. 2009, ApJ, 695, L149

Robbins, W. J., Gaensler, B. M., Murphy, T., Reeves, S., \& Green, A. J. 2012, MNRAS, 419, 2623

Ryter, C., Cesarsky, C. J., \& Audouze, J. 1975, ApJ, 198, 103

Sabbadin, F., Minello, S., \& Bianchini, A. 1977, A\&A, 60, 147

Shaw, R. A., \& Dufour, R. J. 1995, PASP, 107, 896

Stil, J. M., Taylor, A. R., Dickey, J. M., et al. 2006, AJ, 132, 1158

Stupar, M., \& Parker, Q. A. 2011, MNRAS, 414, 2282

Taylor, A. R., Gibson, S. J., Peracaula, M., et al. 2003, AJ, 125, 3145

Trushkin, S. A. 1999, A\&A, 352, L103

Wright, E. L., Eisenhardt, P. R. M., Mainzer, A. K., et al. 2010, AJ, 140, 1868

Zou, W. Z., Hobbs, G., Wang, N., et al. 2005, MNRAS, 362, 1189 